\title{
Trends in paediatric bloodstream infections at a South African referral hospital
}

\author{
Angela Dramowski ${ }^{1 *}$, Mark F Cotton ${ }^{1}$, Helena Rabie ${ }^{1}$ and Andrew Whitelaw ${ }^{2}$
}

\begin{abstract}
Background: The epidemiology of paediatric bloodstream infection (BSI) in Sub-Saharan Africa is poorly documented with limited data on hospital-acquired sepsis, impact of HIV infection, BSI trends and antimicrobial resistance.

Methods: We retrospectively reviewed paediatric BSI (0-14 years) at Tygerberg Children's Hospital between 1 January 2008 and 31 December 2013 (excluding neonatal wards). Laboratory and hospital data were used to determine BSI rates, blood culture contamination, pathogen profile, patient demographics, antimicrobial resistance and factors associated with mortality. Fluconazole resistant Candida species, methicillin-resistant Staphylococcus aureus (MRSA), multi-drug resistant Acinetobacter baumannii and extended-spectrum beta-lactamase (ESBL) producing Enterobacteriaceae were classified as antimicrobial resistant pathogens.
\end{abstract}

Results: Of 17001 blood cultures over 6 years, 935 cultures isolated 979 pathogens (5.5\% yield; 95\% Cl 5.3-5.7\%). Contamination rates were high (6.6\%, 95\% Cl 6.4-6.8\%), increasing over time $(p=0.003)$. Discrete BSI episodes were identified $(n=864)$ with median patient age of 7.5 months, male predominance $(57 \%)$ and $13 \%$ HIV prevalence. BSI rates declined significantly over time (4.6-3.1, overall rate 3.5 per 1000 patient days; 95\% Cl 3.3-3.7; Chi square for trend $p=0.02$ ). Gram negative pathogens predominated (60\% vs $33 \%$ Gram positives and 7\% fungal); Klebsiella pneumoniae (154; 17\%), Staphylococcus aureus $(131 ; 14 \%)$ and Escherichia coli $(97 ; 11 \%)$ were most prevalent. Crude BSI mortality was 20\% (176/864); HIV infection, fungal, Gram negative and hospital-acquired sepsis were significantly associated with mortality on multivariate analysis. Hospital-acquired BSI was common (404/864; 47\%). Overall antimicrobial resistance rates were high ( $70 \%$ in hospital vs $25 \%$ in community-acquired infections; $p<0.0001$ ); hospital-acquired infection, infancy, HIV-infection and Gram negative sepsis were associated with resistance. S. pneumoniae BSI declined significantly over time (58/465 [12.5\%] to 33/399 [8.3\%]; $p=0.04)$.

Conclusion: Although BSI rates declined over time, children with BSI had high mortality and pathogens exhibited substantial antimicrobial resistance in both community and hospital-acquired infections. Blood culture sampling technique and local options for empiric antimicrobial therapy require re-evaluation.

Keywords: Bloodstream infection, Sepsis, Community-acquired infection, Hospital-acquired infection, Healthcare-associated infection, paediatrics, antimicrobial resistance, vaccination, HIV

\section{Background}

The epidemiology of paediatric bloodstream infection (BSI) in Africa is poorly documented. A meta-analysis of prospective studies of community-acquired BSI [1] identified 22 eligible studies (four in Southern Africa) [2-5], where non-typhoidal Salmonella, E. coli, S. aureus and S. pneumoniae infection predominated. Despite published

\footnotetext{
* Correspondence: dramowski@sun.ac.za

'Department of Paediatrics and Child Health, Division of Paediatric Infectious Diseases, Faculty of Medicine and Health Sciences, Stellenbosch University, PO Box 19063, Tygerberg 7505, South Africa

Full list of author information is available at the end of the article
}

descriptions of community-acquired sepsis in African children [1-9] data on hospital-acquired BSI are extremely limited $[10,11]$. It is estimated that healthcareassociated BSI may be responsible for 25000 deaths in African children annually [12]. Overall, incidence rates of healthcare-associated infection in developing countries are thought to be at least double that of high-income settings [13]. Further research on the epidemiology of hospital-acquired BSI in African children is needed to quantify the burden and better understand contributory factors. 
Significant changes in BSI epidemiology among African children are expected, given increasing access to HIV prevention programmes, paediatric antiretroviral therapy and inclusion of pneumococcal conjugate vaccine (PCV) in the immunisation schedule $[14,15]$. In light of globally increasing antimicrobial resistance [12], regional antimicrobial resistance prevalence and the efficacy of empiric antibiotic therapy for paediatric BSI in SubSaharan Africa also require evaluation. Descriptions of increasing prevalence of Enterobacteriaceae BSI isolates producing extended-spectrum beta-lactamases (ESBL) are concerning given the limited availability of appropriate antibiotics in many African countries [16].

The difficulties in obtaining paediatric blood culture specimens and the increased yield with higher volume blood inoculum are well described [8]. Of concern in our region are two recent audits $[17,18]$ reporting low pathogen yields and high blood culture contamination rates (exceeding accepted rates of < 3\%) [19]. We examined trends in paediatric BSI epidemiology over six years at a single academic institution, determining rates of bacteraemia, blood culture yield and contamination, BSI-associated mortality and prevalence of antimicrobial resistance. We also investigated the association between bacteraemia, antimicrobial resistance and HIV infection.

\section{Methods}

\section{Setting}

We retrospectively reviewed paediatric BSI at Tygerberg Children's Hospital (TCH) in Cape Town, South Africa between 1 January 2008 and 31 December 2013 (excluding neonatal wards). The TCH admits sick infants and children $(0-14$ years) requiring general $(70 \%)$ or specialised $(30 \%)$ paediatric care (haematology/oncology, nephrology, gastroenterology, infectious diseases, cardiology, neurology, pulmonology, paediatric surgery, endocrinology) to one of six wards (153 beds; $85 \%$ occupancy rate in 2013). The 10-bedded medical/surgical paediatric intensive care unit (PICU) has an $89 \%$ occupancy rate (2013). Critically-ill children requiring ventilation or inotropic support are preferentially managed in the PICU but also on the wards when PICU is full.

The antenatal HIV prevalence in the Western Cape Province was $16.9 \%$ in 2012 (versus $29.5 \%$ nationally) [20]. Among children aged 2-14 years, HIV prevalence in the Western Cape Province was $0.7 \%$ in 2012 (versus $2.4 \%$ nationally) [21]. Antiretroviral therapy has been available since 2004, including prevention of mother-tochild HIV infection transmission, with transmission rates of $3.9 \%$ in 2010 [22]. HIV testing with informed consent is performed if the child's status is unknown, using an HIV PCR if $<18$ months and HIV Elisa if $>18$ months. If the HIV status is already known, no laboratory sample is submitted. Thus for this study only HIV tests taken at the time of hospitalisation, or taken at prior hospital visits were accessible.

Immunisation coverage in the Cape Metropolitan area in $2011 / 12$ was $87.5 \%$ among the population $<1$ year of age (including BCG, polio, diphtheria/tetanus/pertussis, hepatitis B and measles vaccines; rotavirus was introduced in 2009) [23]. Haemophilus influenzae serotype B (Hib) conjugate vaccine was introduced in 1995 and PCV for $S$. pneumoniae in 2009 (7-valent) and 2011 (13-valent) [15].

\section{Investigation and management of BSI}

Blood cultures are obtained from all children with suspected sepsis or severe infection with a focal site (e.g. pneumonia, cellulitis). Empiric antibiotic therapy for community-acquired sepsis depends on the presumed site of infection, but usually includes either ceftriaxone or ampicillin and gentamicin. Empiric treatment of hospital-acquired infection usually includes meropenem, or ertapenem if Pseudomonas aeruginosa is considered unlikely and meningitis is excluded. Vancomycin is added if MRSA is considered a likely pathogen e.g. with suspected central line sepsis or soft tissue infection in hospital.

\section{Blood culture sampling and analysis}

A single blood culture sample (one bottle) is submitted from most patients, unless infective endocarditis is suspected. Local guidelines recommend inoculation of at least $2 \mathrm{~mL}$ of blood into paediatric blood culture bottles, however for older children, larger blood inoculums of $5-10 \mathrm{ml}$ are encouraged. Blood cultures are taken at the discretion of attending clinicians and transferred to the on-site National Health Laboratory Service (NHLS) microbiology laboratory for processing in an automated system. Prior to April 2011 the Bactec system (Becton Dickinson, New Jersey, United States) was used; and thereafter the BacT/Alert system (BioMerieux, Marcy l' Etoile, France), in line with NHLS policy. For both systems, paediatric-specific culture bottles were used (Becton Dickinson BACTEC Peds Plus/F and thereafter BacT/ ALERT $^{\circ}$ PF bottles). Both contain specialized media that accommodate small-volume samples ( $\leq 3 \mathrm{~mL}$ of blood) and resin for antibiotic neutralization. If bacterial growth is detected in the bottles, a Gram stain is performed, the sample sub-cultured onto appropriate media based on the Gram stain and incubated overnight. Further identification and antimicrobial susceptibility testing of clinically significant isolates is performed with the automated Vitek II system (BioMerieux, Marcy l'Etoile, France), using annually published Clinical and Laboratory Standards Institute (CLSI) breakpoints [24]. Pneumococcal isolates were considered non-susceptible to penicillin at minimum inhibitory concentrations (MICs) $\geq 0.12 \mathrm{mg} / \mathrm{L}$ using the meningitis breakpoints for parenteral penicillin [24]. 


\section{Data analysis}

All positive blood cultures from the paediatric wards over the six year study period were extracted from the computerised laboratory database; demographic data was obtained from the laboratory and hospital admissions database. BSI rates, blood culture contamination, pathogen profile, patient demographics and factors associated with antimicrobial resistance and BSI mortality were determined. Organisms were categorised using the United States Centers for Disease Control (US CDC) list of pathogens and contaminants; common commensals defined by the CDC include diphtheroids [Corynebacterium spp. not C. diphtheriae], Bacillus spp. [not B. anthracis], Propionibacterium spp., coagulase-negative staphylococci [including S. epidermidis], viridans group streptococci, Aerococcus spp., and Micrococcus spp [25]. Positive blood cultures obtained $<72$ hours after admission were classified as community-acquired sepsis. Those obtained $>72$ hours after admission were considered hospital-acquired sepsis. All positive blood cultures isolating the same pathogen within 14 days were considered a single episode of BSI. Fluconazole resistant Candida species, methicillin-resistant Staphylococcus aureus (MRSA), multi-drug resistant Acinetobacter baumannii (resistant to at least 3 classes of antimicrobials) and extended spectrum B-lactamase (ESBL)-producing Enterobacteriaceae were classified as antimicrobial resistant pathogens using proposed standard definitions [26].

\section{Statistical analysis}

The BSI rate was calculated by dividing the total number of BSI episodes by the total inpatient days accumulated during the 6 year period. The pathogen and contamination rates were calculated by dividing the number of blood cultures yielding pathogens and contaminants respectively by the total number of blood culture requested. Continuous and categorical variables were compared using student $\mathrm{t}$ tests and Chi square analysis respectively. A Chi square test for linear trend was used to assess change in rates over the study period. To determine factors associated with mortality from BSI and antimicrobial resistance, binary logistic regression analyses were performed. A p-value below 0.05 was considered statistically significant. Stata Statistical Software version 13.0 IC (College Station, TX: StataCorp LP) was used.

\section{Ethical approval}

Ethical approval and waiver of individual informed consent was obtained from the Human Health Research Ethics committee of Stellenbosch University (S13/09/171).

\section{Results}

For 63209 children hospitalized over the study period, 17001 blood culture specimens were submitted; 1 blood culture per 3.7 admissions or 68.6 specimens/1000 patient days. From 935 culture-positive specimens, 979 BSI pathogens were isolated (5.5\% yield; 95\% CI 5.3$5.7 \%)$. Blood culture contamination rates were high (1123 contaminated blood cultures from 17001 blood culture specimens submitted [6.6\%]; 95\% CI 6.4-6.8\%), increasing over time $(\mathrm{p}=0.003)$ (Figure 1). Coagulasenegative staphylococci (CoNS) were the most commonly isolated contaminant $(650 / 1123 ; 57.9 \%)$, followed by nonpathogenic streptococci $(75 / 1123 ; 6.7 \%)$, Bacillus species (74/1123; 6.6\%), Micrococcus species (63/1123; 5.6\%) and diphtheroids (61/1123; 5.4\%).

Of 864 discrete BSI episodes, 818 (94.7\%) were monomicrobial infections and $46(5.3 \%)$ were polymicrobial (42 with 2 pathogens; 4 with 3 pathogens). The overall BSI rate was 3.5 per 1000 patient days (95\% CI 3.3-3.7). A significant decline in the BSI rate from 4.6 in 2008 to 3.1 in 2013 (Chi square for trend $\mathrm{p}=0.02$; Figure 1) was noted despite substantial increases in hospitalizations (7537 to 11201 [49\%]) and number of blood cultures (7816 vs 9185 [17\%]) submitted between 2008 and 2013.

The median age of patients with BSI episodes was 7.5 months with male predominance $(57.4 \%)$ and $13.4 \%$ HIV prevalence (however the test positivity rate was $20.6 \%$ [116/564], when children of unknown HIV [ $n=299]$ status were excluded) (Table 1). Most patients (679; 78.6\%) had blood cultures submitted from general paediatric wards i.e. were not yet in the PICU. Nearly half of all BSI episodes were hospital-acquired $(404 ; 46.8 \%)$, with a median hospital stay of 17 days (IQR $=8-33.5$ days) before onset of BSI. The overall rate of hospital-acquired BSI was 1.63 episodes per 1000 patient days (404/247969; 95\% CI 1.49 - 1.78) and declined between 2008-2010 and 20112013 (from 1.79 to $1.49 / 1000$ patient days; $\mathrm{p}=0.06$ ). The risk of developing hospital-acquired BSI during hospitalisation was 6.4 per 1000 admissions (95\% CI 5.8 - 7.0).

Gram negative organisms predominated (60.2\%) followed by Gram positives (32.4\%) and fungi (7.4\%). Klebsiella pneumoniae (154; 17\%), Staphylococcus aureus (131; 14\%) and Escherichia coli $(97 ; 11 \%)$ were most prevalent (Table 2). The profile and proportional representation of pathogens varied markedly by location (ward vs PICU) and by place of onset. Gram positive pathogens were more prevalent in community-acquired isolates and Gram negative and fungal pathogens in hospitalacquired sepsis. The spectrum and ranking of BSI pathogens among children known to be HIV infected was similar to that of HIV uninfected patients, except for $S$. pneumoniae which was more common in HIV infected children (20/116 [17.2\%] vs 30/448 [6.7\%]; p < 0.001).

While $H$. influenzae BSI episodes increased between 2008-2010 and 2011-2013 (7/465 [1.5\%] to 15/399 [3.8\%]; $\mathrm{p}=0.05)$, the proportion of $H$. influenzae serotype B isolates remained similar (3/7 [42.9\%] vs 5/15 


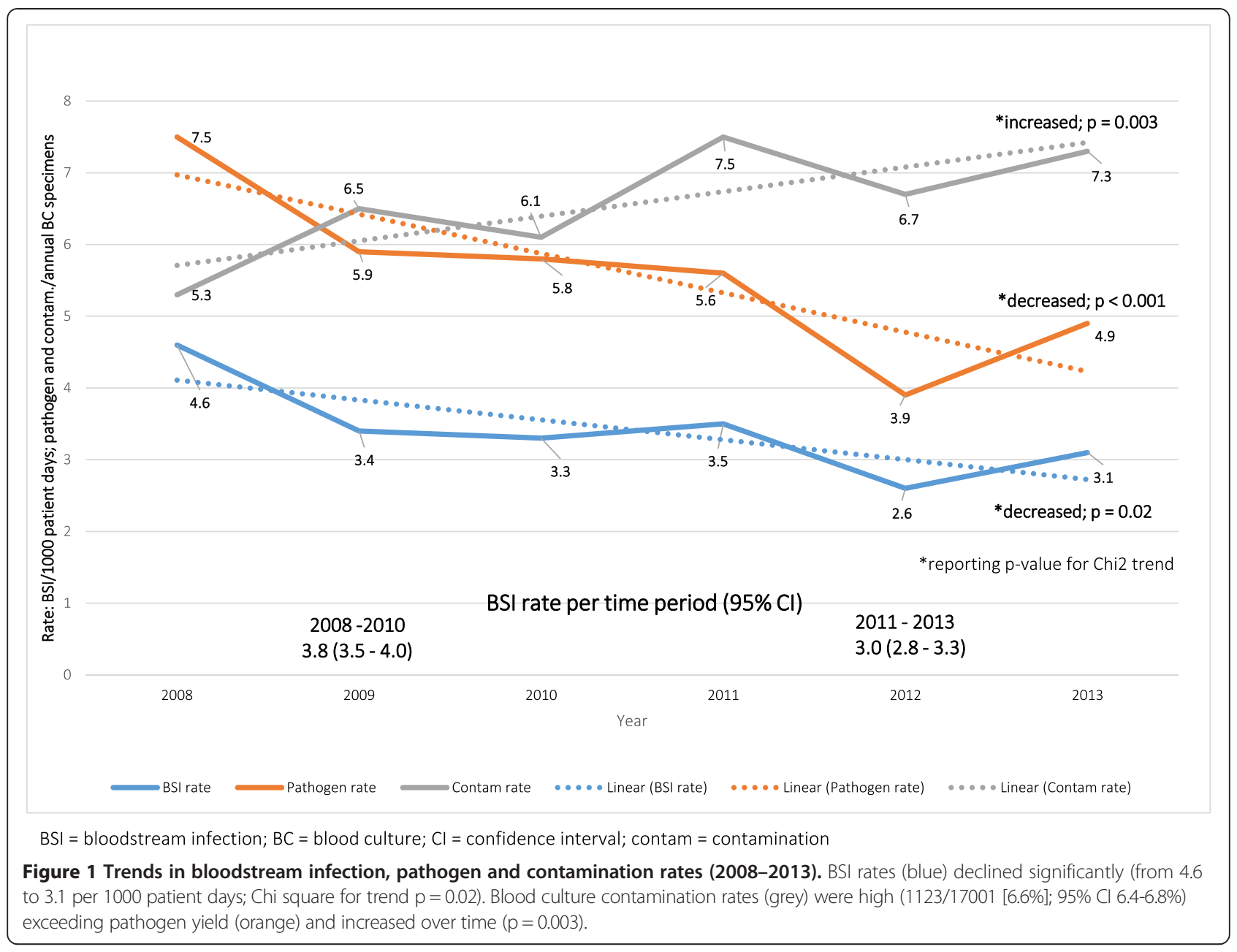

[33.3\%]; p =0.65). However, S. pneumoniae BSI declined significantly $(58 / 465[12.5 \%]$ to $33 / 399[8.3 \%])(\mathrm{p}=0.04)$. Pneumococci as a percentage of all Gram positive pathogens declined from a pre-vaccine high of $43.5 \%(27 / 62)$ in 2008 to $13.1 \%(8 / 61)$ in 2013 [ $\mathrm{p}<0.001$ ]. The proportion of pneumococcal BSI due to vaccine-serotypes (accounting for PCV7 and PCV13) did not decrease significantly over the two time periods, despite PCV-13 giving broader coverage $(20 / 68$ [29.4\%] vs $6 / 23$ [26\%], $\mathrm{p}=0.99)$. The proportion of pneumococcal BSI exhibiting penicillinresistance remained unchanged between 2008-2010 and $2011-2013,(18 / 58$ [31\%] vs $10 / 33$ [30.3\%]; $\mathrm{p}=1.0)$.

Overall crude BSI mortality was 20.4\% (176/864); patients with hospital-acquired BSI experienced higher mortality than community-acquired BSI (25\% [101/404] vs $16.3 \%$ [75/460]; $\mathrm{p}=0.002$ ). The pathogen associated with the highest BSI mortality was Acinetobacter spp $(\mathrm{p}=0.03)$ at $38 \%(30 / 78)$, followed by Candida species (31\%; 20/65) and E coli BSI (24\%; 23/97). HIV, fungi, Gram negative organisms and hospital-acquired sepsis were significantly associated with BSI mortality on multivariate analysis (Table 3 ).
Prevalence of antimicrobial resistance was assessed among a subset of pathogens, focussing on fluconazole resistant Candida species and four bacterial pathogens: MRSA, multi-drug resistant Acinetobacter baumannii and ESBL-producing E coli and K. pneumoniae (Figure 2). No carbapenem resistant Enterobacteriaceae (CRE) or vancomycin resistant Enterococci (VRE) were isolated. For the four selected bacterial pathogens, antimicrobial resistance prevalence among community isolates was $25 \%$ compared with $70 \%$ among hospital-acquired isolates $(\mathrm{p}<0.001)$. For A. baumannii and MRSA, resistance rates were significantly higher among hospital-acquired isolates, whereas $E$. coli and K. pneumoniae isolates had similar prevalence of ESBLs among hospital- and community-acquired isolates. Among the 65 Candida species, all 21 C. albicans isolates were fluconazole susceptible, while 22 of the $44(50 \%)$ non-albicans Candida species were fluconazole resistant. The prevalence of antimicrobial resistance did not differ significantly between 2008-2010 and 2011-2013 for the selected pathogens [Chi square for trend $\mathrm{p}=0.24$ and $\mathrm{p}=0.14$ respectively]. Factors associated with antimicrobial resistance on multivariate analysis included 
Table 1 Demographic profile of paediatric patients with bloodstream infection

\begin{tabular}{|c|c|c|c|c|c|c|}
\hline Variable & Number (n) & Percentage (\%) & $\begin{array}{l}\text { Age } \\
<1 \mathrm{yr} \\
(\mathrm{n} ; \%)\end{array}$ & $\begin{array}{l}\text { Age } \\
1-5 \text { yrs } \\
(n ; \%)\end{array}$ & $\begin{array}{l}\text { Age } \\
5-14 \text { yrs } \\
(n ; \%)\end{array}$ & p-value \\
\hline Laboratory confirmed-BSI episodes* & 864 & $100 \%$ & $506(58.6)$ & $206(23.8)$ & $152(17.6)$ & - \\
\hline \multirow[t]{2}{*}{ Median age (months) } & 7.5 & IQR & 3.2 & 18.2 & 96 & - \\
\hline & & 2.9-23.8 & & & & \\
\hline Male & 496 & 57.4 & $298(58.9)$ & $111(53.9)$ & $89(58.5)$ & 0.41 \\
\hline \multicolumn{7}{|l|}{ HIV status } \\
\hline - Positive & 116 & 13.4 & $68(13)$ & $31(15)$ & $17(11)$ & \\
\hline - Negative & 448 & 52 & $271(54)$ & $101(49)$ & $76(50)$ & 0.57 \\
\hline - Unknown & 299 & 34.6 & $167(33)$ & $74(36)$ & $59(39)$ & \\
\hline \multicolumn{7}{|l|}{ Onset of $\mathrm{BSI}^{\#}$} \\
\hline - Community-acquired & 460 & 53.2 & $244(53)$ & $133(29)$ & $83(18)$ & $<0.001$ \\
\hline - Hospital-acquired & 404 & 46.8 & $262(65)$ & $73(18)$ & $69(17)$ & \\
\hline \multicolumn{7}{|l|}{ Ward at BSI diagnosis } \\
\hline - Intensive care & 185 & 21.4 & $140(28)$ & $33(16)$ & $12(8)$ & $<0.001$ \\
\hline - General ward & 679 & 78.6 & $366(72)$ & $173(84)$ & $140(92)$ & \\
\hline \multicolumn{7}{|l|}{ BSI outcome } \\
\hline - Died & 176 & 20.4 & $109(22)$ & $45(22)$ & $22(14)$ & 0.13 \\
\hline - Survived & 688 & 79.6 & $397(78)$ & $161(78)$ & $130(86)$ & \\
\hline Crude mortality rate by age & - & 20.4 & 21.5 & 21.8 & 14.5 & - \\
\hline
\end{tabular}

*BSI episodes: blood culture sampling episodes that yielded a pathogen, excluding blood cultures that isolated the same organism within 14 days of the original sampling episode; IQR = interquartile range; ${ }^{\#} \mathrm{CA}-\mathrm{BSI}=\mathrm{BC}$ submitted within first 72 hours of admission; $\mathrm{HA}-\mathrm{BSI}=\mathrm{BC}$ submitted $>72$ hours after admission. Continuous and categorical variables were compared using student $t$ tests and Chi square analysis respectively; $\mathrm{p}<0.05$ was considered statistically significant.

hospital-acquired infection, infancy, HIV-infection and Gram negative sepsis (Table 4).

Susceptibility to different combinations of empiric antimicrobial therapy for hospital-acquired BSI was determined for all BSI isolates from 2012 and 2013 (Table 5). The combination of meropenem and amikacin was the most active against both ward and ICU BSI isolates, based on the in-vitro susceptibility test results (overall $82 / 133 ; 83.6 \%$ of isolates susceptible to one or both agents).

\section{Discussion}

In keeping with previous studies from Africa, gram negatives predominated in our study. E. coli and Klebsiella spp. were the most prevalent Enterobacteriaceae. Nontyphoidal salmonellae, a prominent BSI pathogen in malaria-endemic regions [27], was uncommon in our study. S. pneumoniae (the most common isolate in community-acquired BSI in African children [1]), was also prominent in our cohort. In addition, the profile of gram positive isolates changed significantly over time owing to reduced frequency of $S$. pneumoniae detection. The proportion of vaccine-serotype pneumococcal BSI isolates remained stable over time suggesting that the dramatic decline in pneumococcal sepsis rates is not solely attributable to vaccine. However, a study of invasive pneumococcal disease in South Africa did demonstrate a substantial reduction of $89 \%$ in disease caused by PCV7 serotypes in children $<2$ years old [28]. Other factors such as increasing antiretroviral uptake and decreasing HIV prevalence probably contributed. Fungi (especially hospital-acquired) and gram negatives were significantly associated with BSI mortality, as in other African studies $[6,10,11]$.

The burden of paediatric BSI was concentrated among infants (58.6\% of the cohort). Young age was not associated with mortality from BSI but was significantly associated with antimicrobial resistant pathogens. Overall BSI mortality in our cohort (20.4\%) was higher than that reported for high income settings (11 - 14\%) [29,30], but lower than other African settings $(27-38 \%)[6,10,11]$. Access to intensive care was not reported for the other African BSI studies and likely contributed to the lower case fatality in our cohort, although PICU facilities at our institution are very limited (only 1 in 5 patients was admitted in PICU at BSI diagnosis).

As previously reported [10], HIV-infected children were at increased risk for BSI-associated mortality and 
Table 2 Microbiological profile of paediatric bloodstream infection episodes $(n=864)$

\begin{tabular}{|c|c|c|c|c|}
\hline BSI episodes & $\mathbf{n}$ & $\%$ & \multicolumn{2}{|c|}{ Total pathogens isolated from 864 BSI episodes } \\
\hline Monomicrobial & 818 & 94.7 & 914 & \\
\hline \multicolumn{5}{|l|}{ Polymicrobial } \\
\hline - 2 pathogens & 42 & 4.8 & & \\
\hline - 3 pathogens & 4 & 0.5 & & \\
\hline Gram negatives & Organism & $\mathrm{n}=550$ & $\%$ of Gram negatives & Organism rank* \\
\hline \multirow[t]{9}{*}{ Enterobacteriaceae } & K. pneumoniae & 154 & $28 \%$ & 1 \\
\hline & E. coli & 97 & $18 \%$ & 3 \\
\hline & E. cloacae & 30 & $5 \%$ & 7 \\
\hline & S. marcescens & 19 & $3 \%$ & 10 \\
\hline & Salmonella spp & 18 & $3 \%$ & \\
\hline & (non-typhi) & & & \\
\hline & Klebsiella spp & 12 & $2 \%$ & \\
\hline & Other (9 different genera) & 30 & $5 \%$ & \\
\hline & Total & 360 & $65 \%$ & \\
\hline \multirow[t]{6}{*}{ Non-fermenting Gram negative bacilli } & A. baumannii & 78 & $14 \%$ & 5 \\
\hline & P. aeruginosa & 20 & $4 \%$ & 9 \\
\hline & Acinetobacter spp & 16 & $3 \%$ & \\
\hline & S. paucimobilis & 9 & $2 \%$ & \\
\hline & Other (9 different genera) & 20 & $4 \%$ & \\
\hline & Total & 143 & $26 \%$ & \\
\hline \multirow[t]{4}{*}{ Other Gram negative organisms } & H. influenzae & 23 & $4 \%$ & 8 \\
\hline & N. meningitidis & 11 & $2 \%$ & \\
\hline & Other (6 different genera) & 13 & $3 \%$ & \\
\hline & Total & 47 & $9 \%$ & \\
\hline Gram positives & Organism & $n=296$ & $\%$ of Gram positives & Organism rank* \\
\hline Staphlylococci & Staphylococcus aureus & 131 & $44 \%$ & 2 \\
\hline \multirow[t]{2}{*}{ Streptococci } & Streptococcus pneumoniae & 91 & $31 \%$ & 4 \\
\hline & Group B Streptococcus & 19 & $6 \%$ & 10 \\
\hline Enterococci & Enterococcus spp & 46 & $16 \%$ & 6 \\
\hline Other Gram positive organisms & Other (4 different genera) & 9 & $3 \%$ & \\
\hline Fungi & Organism & $n=68$ & $\%$ of Fungi & Organism rank* \\
\hline \multirow[t]{6}{*}{ Candida spp } & Candida albicans & 21 & $31 \%$ & 9 \\
\hline & Candida tropicalis & 12 & $18 \%$ & \\
\hline & Candida parapsilosis & 9 & $13 \%$ & \\
\hline & Candida glabrata & 3 & $4 \%$ & \\
\hline & Candida krusei & 2 & $3 \%$ & \\
\hline & All other candida spp & 18 & $27 \%$ & \\
\hline \multirow[t]{3}{*}{ Other fungi } & Aspergillus spp, & 1 & $4 \%$ & \\
\hline & Trichosporon spp, & 1 & & \\
\hline & unidentified yeast & 1 & & \\
\hline
\end{tabular}


Table 2 Microbiological profile of paediatric bloodstream infection episodes $(\mathbf{n}=\mathbf{8 6 4})$ (Continued)

BSI pathogens $(n=914)$ by type and place of infection onset for 864 BSI episodes

Community-acquired (CA-BSI) pathogens

$\mathrm{n}=\mathbf{4 7 7}$

CA-BSI ward pathogens"

$n=433$

- Staphylococcus aureus

- Streptococcus pneumoniae

- Escherichia coli

- Other

CA-BSI ICU pathogens ${ }^{\#}$

- Escherichia coli

- Klebsiella pneumoniae

- Staphylococcus aureus

- Other
Hospital-acquired (HA-BSI) pathogens

$n=437$

HA-BSI ward pathogens ${ }^{\#} \quad \mathrm{n}=275 \quad \%$

- Klebsiella pneumoniae 86

- Candida spp 28

- Acinetobacter baumanni 26

- Other 135

HA-BSI ICU pathogens ${ }^{\#} \quad \mathrm{n}=162$

- Acinetobacter baumanni

42

- Klebsiella pneumoniae

33

- Candida spp

22

- Other

${ }^{*}$ Total pathogens isolated from $864 \mathrm{BSI}$ episodes $=914$ pathogens ( 818 monomicrobial + polymicrobial $42 \times 2$ isolates $+4 \times 3$ isolates) ${ }^{\#}$ CA-BSI $=\mathrm{BC}$ submitted within first 72 hours of admission; HA-BSI $=B C$ submitted $>72$ hours after admission; ICU = intensive care unit; * Organism rank reported for the top ten isolates only.

Table 3 Bloodstream infection-associated mortality

\begin{tabular}{|c|c|c|c|c|}
\hline BSI-associated mortality & Number (n) & Percentage (\%) & p-value & \\
\hline Total BSI-associated deaths & 176 & 100 & - & \\
\hline Male & 97 & 55.1 & 0.49 & \\
\hline Median age (months) IQR & 7.2 & IQR 3-14.7 & - & \\
\hline \multicolumn{5}{|l|}{ HIV status } \\
\hline - Positive & 34 & 19.3 & & \\
\hline - Negative & 87 & 49.4 & 0.03 & \\
\hline - Unknown & 55 & 31.3 & & \\
\hline \multicolumn{5}{|l|}{ Onset of $\mathrm{BSI}^{\#}$} \\
\hline - Community-acquired & $75 / 460$ & 16.3 & 0.002 & \\
\hline - Hospital-acquired & $101 / 404$ & 25 & & \\
\hline \multicolumn{5}{|c|}{ Factors associated with mortality from BSI } \\
\hline Variable assessed & $\begin{array}{l}\text { Univariate analysis } \\
\text { (p-value) }\end{array}$ & $\begin{array}{l}\text { Multivariate analysis } \\
\text { (p-value) }\end{array}$ & Odds ratio & $95 \% \mathrm{Cl}$ \\
\hline Length of stay prior to BSI onset & $<0.001$ & 0.11 & - & - \\
\hline Age category & 0.13 & 0.44 & - & - \\
\hline Gender & 0.49 & 0.32 & - & - \\
\hline HIV status (positive) & 0.03 & 0.02 & 1.74 & $1.1-2.8$ \\
\hline Year of BSI & 0.89 & 0.78 & - & - \\
\hline Place of BSI onset (hospital-acquired) & 0.002 & 0.04 & 1.43 & $1.1-2.0$ \\
\hline Type of BSI pathogen & 0.001 & 0.03 & & \\
\hline - Fungal & & & 2.10 & $1.1-4.2$ \\
\hline - Gram negative & & & 1.88 & $1.2-2.9$ \\
\hline Mono- vs poly-microbial BSI & 0.72 & 0.6 & - & - \\
\hline ICU vs general ward at BSI onset & $<0.001$ & 0.001 & 2.93 & $1.9-4.4$ \\
\hline Antimicrobial resistance & 0.06 & 0.83 & - & - \\
\hline
\end{tabular}

$\mathrm{BSI}=$ bloodstream infection; $\mathrm{ICU}=$ intensive care unit; ${ }^{\#} \mathrm{CA}-\mathrm{BSI}=\mathrm{BC}$ submitted within first 72 hours of admission; $\mathrm{HA}-\mathrm{BSI}=\mathrm{BC}$ submitted $>72$ hours after admission. To determine factors associated with mortality from BSI and antimicrobial resistance, binary logistic regression analyses were performed. A p-value below 0.05 was considered statistically significant. 


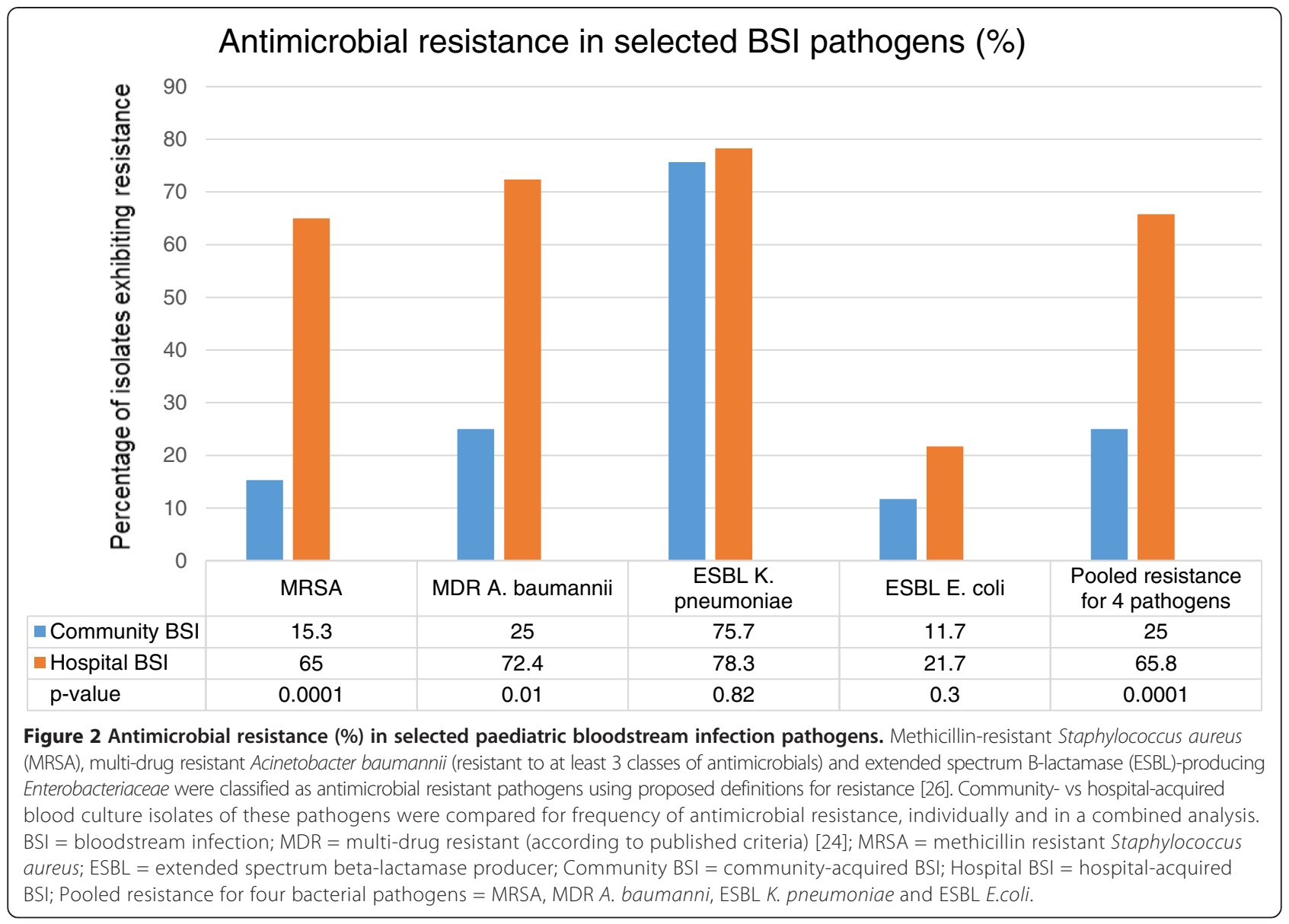

more likely to have antimicrobial resistant pathogens. Bacterial colonisation is a risk factor for later invasive infection with high rates of colonisation with antimicrobial resistant pathogens described among HIV-infected children in Cape Town [31]. It was not possible (given the study design) to compare the relative risk for BSI among HIV-infected versus HIV-uninfected children or the effect of antiretroviral therapy. A prospective study is underway at our institution to determine the relative risk for hospital-acquired infection (including BSI) among HIV-infected children.

Hospital-acquired BSI was common (nearly half of all BSI episodes), more prevalent among infants and significantly associated with mortality. The profile of hospital-acquired

Table 4 Factors associated with antimicrobial resistance

\begin{tabular}{|c|c|c|c|c|}
\hline Variable assessed & $\begin{array}{l}\text { Univariate analysis } \\
\text { (p-value) }\end{array}$ & $\begin{array}{l}\text { Multivariate analysis } \\
\text { (p-value) }\end{array}$ & Odds ratio & $95 \% \mathrm{Cl}$ \\
\hline Length of stay prior to BSI onset & $<0.001$ & 0.53 & - & - \\
\hline Age category (infants) & $<0.001$ & 0.003 & 1.92 & $1.2-3.1$ \\
\hline Gender & 0.8 & 0.92 & - & - \\
\hline HIV status (positive) & $<0.001$ & $<0.001$ & 2.64 & $1.7-4.2$ \\
\hline Year of BSI & 0.4 & 0.19 & - & - \\
\hline Place of BSI onset (hospital-acquired) & $<0.001$ & $<0.001$ & 3.68 & $2.7-5.1$ \\
\hline \multicolumn{5}{|l|}{ Type of BSI pathogen } \\
\hline - Gram negative & $<0.001$ & $<0.001$ & 1.99 & $1.4-2.9$ \\
\hline Mono- vs poly-microbial BSI & 0.18 & 0.84 & - & - \\
\hline ICU vs general ward at BSI onset & $<0.001$ & 0.06 & - & - \\
\hline
\end{tabular}

$\mathrm{BSI}=$ bloodstream infection; $\mathrm{ICU}=$ intensive care unit; Hospital-acquired BSI = BC submitted $>72$ hours after admission. To determine factors associated with antimicrobial resistance, binary logistic regression analyses were performed. A p-value below 0.05 was considered statistically significant. 
Table 5 Coverage achieved for hospital-acquired bloodstream infections with empiric antimicrobial regimens (2012-2013)*

\begin{tabular}{|c|c|c|c|c|c|c|c|}
\hline \multirow[t]{3}{*}{ Antibiotic susceptibility } & \multicolumn{2}{|c|}{$\begin{array}{c}\text { OVERALL } \\
n=159\end{array}$} & \multicolumn{2}{|c|}{$\begin{array}{l}\text { WARD } \\
\mathrm{n}=92\end{array}$} & \multicolumn{2}{|c|}{$\begin{array}{c}\text { PICU } \\
n=67\end{array}$} & \multirow{3}{*}{$\begin{array}{l}\text { Ward vs } \\
\text { PICU } \\
\text { p-value }\end{array}$} \\
\hline & \multicolumn{6}{|l|}{ SUSCEPTIBLE } & \\
\hline & Number (n) & Percentage (\%) & Number (n) & Percentage (\%) & Number (n) & Percentage (\%) & \\
\hline piperacillin tazobactam + amikacin & 122 & 76.7 & 71 & 77.2 & 50 & 74.6 & 0.7 \\
\hline ertapenem & 110 & 69.1 & 71 & 77.2 & 40 & 59.7 & 0.02 \\
\hline meropenem & 112 & 70.4 & 70 & 76 & 42 & 62.7 & 0.08 \\
\hline meropenem + amikacin & 133 & 83.6 & 82 & 89.1 & 51 & 76 & 0.03 \\
\hline meropenem + vancomycin & 119 & 74.8 & 73 & 79.3 & 46 & 68.7 & 0.14 \\
\hline
\end{tabular}

$\mathrm{PICU}=$ paediatric intensive care unit; *only pathogens isolated in 2012 and 2013 were included in this analysis in order to determine recent antimicrobial resistance patterns.

pathogens was distinct from that of community-acquired BSI with Klebsiella, Acinetobacter and Candida species predominating. In keeping with the only prospective study of hospital-acquired bacteraemia in African children, Acinetobacter sepsis had the highest case fatality rates in our cohort. The rate of hospital-acquired BSI in our study exceeded the rate of nosocomial bacteraemia in rural Kenya (1.63 versus 1.0 episodes per 1000 patient days), however our cohort had a substantially higher HIV prevalence (13.4\% vs $2 \%$ ) [11]. Unsurprisingly, hospital isolates exhibited significantly greater antimicrobial resistance. Unlike the Tanzanian cohort [10], antimicrobial resistance was not associated with BSI mortality, possibly due to our use of carbapenems for empiric treatment of hospital-acquired sepsis.

A worrying observation is the high rate of antimicrobial resistance among community-acquired pathogens, especially. E.coli and Klebsiella spp. Inappropriate empiric antimicrobial therapy (due to ESBL-producing and multiresistant pathogens) predicted death in the Tanzanian cohort (OR 12.9) [10]. It is possible that pre-hospital antibiotic administration in our cohort may have falsely elevated antimicrobial resistance rates for communityacquired pathogens, by decreasing the frequency of isolation of susceptible pathogens. However our data are in keeping with pooled laboratory data (2010-2012) from public sector hospitals in South Africa demonstrating ESBL-carriage in $68 \%$ of $2774 \mathrm{~K}$. pneumoniae BSI isolates [32]. Ongoing surveillance of antimicrobial resistance in community BSI (and monitoring of clinical outcomes among children given ineffective antibiotic therapy) is needed to determine if antibiotic guidelines need revision.

Among hospital-acquired BSI in the last two years of the study period, isolates exhibited highest susceptibility $(83.6 \%)$ to meropenem plus amikacin, not currently our recommended empiric regimen. A prospective review, which includes clinical data on response to therapy is urgently needed to inform our guidelines for treatment of hospital-acquired sepsis. However, given the need for antimicrobial stewardship (and restriction of carbapenem use), it is also important to assess the efficacy of narrower- spectrum regimens (such as piperacillin-tazobactam and amikacin).

Our review of temporal trends in paediatric BSI epidemiology identified targets for quality improvement. Although not unique to our setting, blood culture contamination rates were high (double the international norm [19]) and increased over time (exceeding the rate of pathogen isolation). The annual pathogen yield declined significantly over time and in 2013 (4.9\%; 95\% CI 3.9 - 5.2) was substantially lower than blood culture yields from a systematic review in Africa (8.2\%; 95\% CI 7.9 - 8.4) [1]. There are several explanations for these findings including poor aseptic technique during specimen collection with failure to isolate pathogens because of overgrowth by c ontaminants, low sensitivity of paediatric blood cultures and sub-optimal blood volumes from children [8]. Prior administration of antibiotics also contributes to low blood culture yield: local management guidelines [33] recommend that criticallyill children referred in from primary care receive a single dose of intramuscular ceftriaxone prior to transfer.

Between the two study time periods, BSI rates declined significantly despite a substantial increase in hospitalization (measured by increased inpatient days) and despite an increase in actual numbers of blood culture specimens submitted. We suspect that improvements in PMTCT programmes, paediatric antiretroviral coverage and $\mathrm{PCV}$ introduction between 2008 and 2013 are partly responsible. However, declining pathogen yields (as described above) may also have artificially reduced BSI rates.

This study has several limitations, most importantly the possibility that some healthcare-associated BSI (readmission within 30 days of hospital discharge) may have been misclassified as community-acquired, owing to the retrospective study design. We chose 72 hours as a more conservative cut-off for hospital-acquired bacteraemia (many authorities use 48 hours) to avoid possibly including some community bacteraemias as hospitalacquired. The time of blood culture collection and the time of developing symptoms/signs of infection (as opposed to blood culture collection) were not routinely 
available. The use of 72 hours as a cut-off, may have slightly underestimated the nosocomial bacteraemia rate. We were unable to evaluate the appropriateness of empiric therapy for community-acquired BSI, as the locally recommended treatment regimen depends on the child's clinical presentation. Lack of standardized patient selection or technique for blood culture, lack of comprehensive clinical data or information on recent antibiotic use and/or hospitalisation are also limitations. Many patients had unknown HIV status, but were likely tested at a different facility.

Although BSI rates declined over time, we could not determine which factors and practices contributed to this trend. In addition, the BSI rates may have been significantly underestimated owing to pre-hospital antibiotic administration and inadequate blood volumes submitted for culture. We believe that change in the laboratory culturing system and average volume of blood inoculum are less likely explanations for the decline in BSI rate, since the blood culture contamination rate increased significantly over time. However, differences in pathogen yield between the two systems have been described, and the impact of a different blood culture system on the BSI rate cannot be completely discounted [34-36].

Given the relatively good resources and care at our institution (including an infection prevention and control service and PICU facilities), these findings may be more generalizable to better-resourced African settings. Recommendations for local practice arising from this study include urgent review of paediatric blood culture practice (emphasizing aseptic technique and adequate blood inoculum) and review of empiric antibiotic therapy for both community and hospital-acquired BSI.

\section{Conclusions}

Children with BSI experienced high mortality, particularly for hospital-acquired infection. S. pneumoniae BSI declined after introduction of $\mathrm{PCV}$ and increasing antiretroviral coverage. Pathogens (both community- and hospitalacquired) exhibited substantial antimicrobial resistance. Although BSI rates declined, blood culture contamination rates increased; blood culture sampling technique and local options for empiric antimicrobial therapy require re-evaluation.

\begin{abstract}
Abbreviations
BSI: Bloodstream infection; Cl: Confidence interval; ESBL: Extended-spectrum beta-lactamase; HIV: Human immunodeficiency virus; MRSA: Methicillin-resistant Staphylococcus aureus; PCV: Pneumococcal conjugate vaccine; PICU: Paediatric intensive care unit; PMTCT: Prevention of mother to child transmission of HIV.
\end{abstract}

\section{Competing interests}

The authors declare that they have no competing interests.

\section{Authors' contributions}

All authors (AD, MC, HR, AW) contributed to study design and critical review of the manuscript. AD carried out the data collection, data cleaning (assisted by AW) and statistical analysis. All authors read and approved the final manuscript.

\section{Acknowledgements}

The primary author (AD) is supported by the Medical Research Council (MRC) Clinician Researcher Programme and the Discovery Foundation Academic Fellowship (but these funding bodies played no role in the design, data collection, data analysis and interpretation, or writing of the manuscript). The authors wish to thank Tonya Esterhuizen from Stellenbosch University's Biostatistics Unit for assistance with data analysis and Dr Jean Maritz from Stellenbosch University's Medical Virology Department for assistance with extraction of HIV results.

\section{Author details}

${ }^{1}$ Department of Paediatrics and Child Health, Division of Paediatric Infectious Diseases, Faculty of Medicine and Health Sciences, Stellenbosch University, PO Box 19063, Tygerberg 7505, South Africa. ${ }^{2}$ Department of Medical Microbiology, Stellenbosch University and the National Health Laboratory Service (NHLS), Cape Town, South Africa.

Received: 21 October 2014 Accepted: 20 March 2015

Published online: 02 April 2015

\section{References}

1. Reddy EA, Shaw AV, Crump JA. Community-acquired bloodstream infections in Africa: a systematic review and meta-analysis. Lancet Infect Dis. 2010;10(6):417-32.

2. Archibald LK, Kazembe PN, Nwanyanwu O, Mwansambo C, Reller LB, Jarvis WR. Epidemiology of bloodstream infections in a bacille Calmette-Guerinvaccinated pediatric population in Malawi. J Infect Dis. 2003;188:202-8.

3. Archibald LK, Nwanyanwu O, Kazembe PN, Mwansambo C, Bell M, Dobbie H, et al. Detection of bloodstream pathogens in a bacille Calmette-Guerin (BCG)-vaccinated pediatric population in Malawi: a pilot study. Clin Microbiol Infect. 2003:9:234-8.

4. Nathoo KJ, Chigonde S, Nhembe M, Ali MH, Mason PR. Community-acquired bacteremia in human immunodeficiency virus-infected children in Harare, Zimbabwe. Pediatr Infect Dis J. 1996;15:1092-7.

5. Sigaúque B, Roca A, Mandomando I, Morais L, Quintó L, Sacarlal J, et al. Community-acquired bacteremia among children admitted to a rural hospital in Mozambique. Pediatr Infect Dis J. 2009;28:108-13.

6. Walsh AL, Phiri AJ, Graham SM, Molyneux EM, Molyneux ME. Bacteremia in febrile Malawian children: clinical and microbiologic features. Pediatr Infect Dis J. 2000;19(4):312-8

7. Cotton MF, Burger PJ, Bodenstein WJ. Bacteraemia in children in the south-western Cape. A hospital-based survey. S Afr Med J. 1992;81(2):87-90.

8. Berkley JA, Lowe BS, Mwangi I, Williams T, Bauni E, Mwarumba S, et al. Bacteremia among children admitted to a rural hospital in Kenya. N Engl J Med. 2005;352(1):39-47.

9. Obaro S, Lawson L, Essen U, Ibrahim K, Brooks K, Otuneye A, et al. Community acquired bacteremia in young children from central Nigeria-a pilot study. BMC Infect Dis. 2011;11:137.

10. Blomberg B, Manji KP, Urassa WK, Tamim BS, Mwakagile DS, Jureen R, et al. Antimicrobial resistance predicts death in Tanzanian children with bloodstream infections: a prospective cohort study. BMC Infect Dis. 2007;7:43.

11. Aiken AM, Mturi N, Njuguna P, Mohammed S, Berkley JA, Mwangi I, et al. Risk and causes of paediatric hospital-acquired bacteraemia in Kilifi District Hospital, Kenya: a prospective cohort study. Lancet. 2011;378(9808):2021-7.

12. Aiken AM, Allegranzi B, Scott JA, Mehtar S, Pittet D, Grundmann H. Antibiotic resistance needs global solutions. Lancet Infect Dis. 2014;14(7):550-1.

13. Allegranzi B, Bagheri Nejad S, Combescure C, Graafmans W, Attar $\mathrm{H}_{4}$ Donaldson $L$, et al. Burden of endemic health-care-associated infection in developing countries: systematic review and meta-analysis. Lancet 2011;377(9761):228-41

14. Musiime V, Cook A, Bakeera-Kitaka S, Vhembo T, Lutakome J, Keishanyu R, et al. Bacteremia, causative agents and antimicrobial susceptibility among HIV-1-infected children on antiretroviral therapy in Uganda and Zimbabwe. Pediatr Infect Dis J. 2013;32(8):856-62.

15. Madhi SA, Bamford L, Ngcobo N. Effectiveness of pneumococcal conjugate vaccine and rotavirus vaccine introduction into the South African public immunisation programme. S Afr Med J. 2014;104(3 Suppl 1):228-34.

16. Tansarli GS1, Poulikakos P, Kapaskelis A, Falagas ME. Proportion of extendedspectrum $\beta$-lactamase (ESBL)-producing isolates among Enterobacteriaceae in Africa: evaluation of the evidence-systematic review. J Antimicrob Chemother. 2014;69(5):1177-84. 
17. Lochan H1, Bamford C, Eley B. Blood cultures in sick children. S Afr Med J. 2013;103(12):918-20.

18. Kenyon CR, Fatti G, Schrueder N, Bonorchis K, Meintjes G. The value of blood culture audits at peripheral hospitals. S Afr Med J. 2012;102(4):224-5.

19. Hall KK, Lyman JA. Updated review of blood culture contamination. Clin Microbiol Rev. 2006;19(4):788-802.

20. National Department of Health South Africa: Pretoria. 2012 National Antenatal Sentinel HIV \& Syphilis Prevalence Survey http://www.health.gov. za/docs/reports/2013/report2014.pdf

21. Shisana O, Rehle T, Simbayi LC, Zuma K, Jooste S, Zungu N, et al. South African National HIV prevalence, incidence and behaviour survey, 2012. Cape Town: HSRC Press; 2014.

22. Goga AE, Dinh TH, Jackson DJ for the SAPMTCTE study group. Evaluation of the Effectiveness of the National Prevention of Mother-to-Child Transmission (PMTCT) Programme Measured at Six Weeks Postpartum in South Africa, 2010 http://www.mrc.ac.za/healthsystems/SAPMTCTE2010.pdf

23. Western Cape Government Provincial Treasury. Municipal economic review \& outlook - Cape Metropolitan Area 2012. http:/www.westerncape.gov.za/assets/ departments/treasury/dc00_mero_2012_city_of_cape_town_metropolitan.pdf]

24. Clinical and Laboratory Standards Institute. Performance standards for antimicrobial susceptibility testing; twenty-first informational supplement; M100-S21. Wayne, PA: Clinical and Laboratory Standards Institute; 2011.

25. Centers for Disease Control and Prevention, and the National Healthcare Safety Network. CDC/NHSH Bloodstream Infection Event (Central LineAssociated Bloodstream Infection and Non-central line-associated Bloodstream Infection) 2015. www.cdc.gov/nhsn/PDFs/pscManual/4PSC_CLABScurrent.pdf and http:/www.cdc.gov/nhsn/XLS/Common-Skin-Contaminant-List-June-2011.xlsX

26. Magiorakos AP, Srinivasan A, Carey RB, Carmeli Y, Falagas ME, Giske CG, et al. Multidrug-resistant, extensively drug-resistant and pandrug-resistant bacteria: an international expert proposal for interim standard definitions for acquired resistance. Clin Microbiol Infect. 2012;18(3):268-81.

27. Graham SM. Nontyphoidal salmonellosis in Africa. Curr Opin Infect Dis, 2010;23(5):409-14.

28. von Gottberg A, de Gouveia L, Tempia S, Quan V, Meiring S, von Mollendorf C, et al. Effects of vaccination on invasive pneumococcal disease in South Africa. N Engl J Med. 2014;371(20):1889-99.

29. Abo-Shadi MA, Al-Johani AA, Bahashwan AA. Antimicrobial resistance in pathogens causing pediatrics bloodstream infections in a Saudi Hospital. British Microbiology Research Journal. 2012;2(4).

30. Wisplinghoff $H$, Seifert $H$, Tallent SM, Bischoff T, Wenzel RP, Edmond MB, Nosocomial bloodstream infections in pediatric patients in United States hospitals: epidemiology, clinical features and susceptibilities. Pediatr Infect Dis J. 2003;22(8):686-91.

31. Cotton MF, Wasserman E, Smit J, Whitelaw A, Zar HJ. High incidence of antimicrobial resistant organisms including extended spectrum beta-lactamase producing Enterobacteriaceae and methicillin-resistant Staphylococcus aureus in nasopharyngeal and blood isolates of HIV-infected children from Cape Town, South Africa. BMC Infect Dis. 2008:8:40.

32. Perovic O, Singh-Moodley A, Dusé A, Bamford C, Elliott G, Swe-Han KS, et al. National sentinel site surveillance for antimicrobial resistance in Klebsiella pneumoniae isolates in South Africa, 2010-2012. S Afr Med J. 2014;104(8):563-8.

33. South African Department of Health. Integrated management of childhood illness guidelines 2011. http://www.rudasa.org.za/index.php/resources/ document-library/category/6-paediatrics?download=29:integratedmanagement-of-childhood-illness-booklet.

34. Sullivan KV, Turner NN, Lancaster DP, Shah AR, Chandler $L$, Friedman DF, et al. Superior sensitivity and decreased time to detection with the Bactec Peds Plus/F system compared to the BacT/Alert Pediatric FAN blood culture system. J Clin Microbiol. 2013:51(12):4083-6.

35. Akan OA, Yildiz E. Comparison of the effect of delayed entry into 2 different blood culture systems (BACTEC 9240 and BaCT/ALERT 3D) on culture positivity. Diagn Microbiol Infect Dis. 2006;54(3):193-6.

36. Zadroga R, Williams DN, Gottschall R, Hanson K, Nordberg V, Deike M, et al. Comparison of 2 blood culture media shows significant differences in bacterial recovery for patients on antimicrobial therapy. Clin Infect Dis. 2013;56(6):790-7.

\section{Submit your next manuscript to BioMed Central and take full advantage of:}

- Convenient online submission

- Thorough peer review

- No space constraints or color figure charges

- Immediate publication on acceptance

- Inclusion in PubMed, CAS, Scopus and Google Scholar

- Research which is freely available for redistribution 